\title{
Púrpura trombocitopénica trombótica en un paciente con infección por VIH
}

\author{
Thrombotic thrombocytopenic purpura, in a HIV-infected patient. Case report
}

Juan Camilo Motta', Camilo Rodríguez', Antonia Pino Marín y Edgar Barrera²

'Universidad del Rosario, Bogotá, Colombia.

${ }^{2}$ Fundación Cardio-infantil, Bogotá, Colombia.

Sin financiamiento

Los autores declaran no tener conflictos de intereses

Recibido: 30 de mayo de 2020 / Aceptado: 9 de noviembre de 2020

\section{Resumen}

Las manifestaciones hematológicas de la infección por el VIH son frecuentes y variadas debido a su capacidad de afectar prácticamente todas las líneas celulares. Dentro de éstas, la púrpura trombocitopénica trombótica (PTT) es una de las entidades que constituyen las microangiopatías trombóticas. Se caracteriza por la presencia de trombocitopenia y anemia hemolítica microangiopática con alteración de la función renal. Actualmente, la co-existencia de estas dos entidades es poco frecuente debido a la terapia anti-retroviral de alta efectividad (TARV) Presentamos el caso de un paciente de 28 años, quien consultó por fiebre asociada a episodios de gingivorragia, palidez mucocutánea generalizada y debilidad progresiva. Los estudios evidenciaron una anemia y trombocitopenia grave. Se encontraron esquistocitos y microesferocitos en el frotis de sangre periférica con actividad de la enzima ADAMTS 13 disminuida (6,8\%). Se confirmó el diagnóstico de una PTT como manifestación inicial de una infección por VIH. Se indicó manejo con plasmaféresis e inicio de TARV con buena respuesta.

Palabras clave: Microangiopatía trombótica; VIH; anemia; trombocitopenia, púrpura trombocitopénica trombótica.

\section{Introducción}

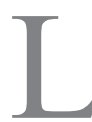

a púrpura trombocitopénica trombótica (PTT) fue descrita por primera vez en 1924 por el Dr. Eli Moschcowitz en una mujer de 16 años, quien presentó petequias, hematuria y alteración neurológica, con documentación post-mortem de trombos diseminados en arteriolas y capilares ${ }^{1}$. Esta patología clasifica dentro de las microan-

\begin{abstract}
Hematological manifestations for human immunodeficiency virus (HIV) infection are frequent and diverse due to its ability to affect almost all cell lines. Among these, thrombotic thrombocytopenic purpura (TTP) is one of the thrombotic microangiopathies syndromes, characterized by the presence of thrombocytopenia and microangiopathic hemolytic anemia with impaired renal function. Nowadays, the relationship between these two entities is rare given the current highly active antiretroviral therapy (HAART). We report the case of a 28-year-old patient, who presented with fever associated with gingival bleeding, generalized mucocutaneous pallor and progressive weakness. Routine investigations showed anemia and severe thrombocytopenia, schistocytes and micro spherocytes in peripheral blood smear. Required blood transfusion, with decreased ADAMTS 13 enzyme activity (6.8\%). With these findings, TTP was diagnosed as the initial manifestation of the HIV infection. The patient received management with five sessions of plasmapheresis and HAART with subsequent improvement.

Keywords: Thrombotic microangiopathy; HIV; anemia; thrombocytopenia; thrombotic thrombocytopenic purpura.
\end{abstract}


penia intensa, anemia hemolítica microangiopática con esquistocitos en frotis de sangre periférica y compromiso de órganos por isquemia en grado variable, con afectación predominante del sistema nervioso central. La pentada clásica de la enfermedad se presenta en menos de $10 \%$ de los $\operatorname{casos}^{3,4}$.

La PTT adquirida tiene una incidencia anual de 2 a 3 casos por cada millón de habitantes y una prevalencia de aproximadamente 13 casos por cada millón de personas. Tiene una alta letalidad en caso de no recibir tratamiento ${ }^{5,6}$. El $90 \%$ de los casos ocurren en la edad adulta, con una edad media de 43 años, con un predominio en mujeres (razón de 2:1) y pacientes de origen afro-descendiente $e^{5,7,8}$. Este desorden puede ser de origen genético o adquirido, por inhibición de la enzima ADAMTS 13 mediada por auto-anticuerpos ${ }^{2,9}$.

La PTT se produce por una deficiencia en la actividad de la enzima ADAMTS 13, una metalo-proteinasa encargada de la escisión del factor de Von Willebrand $(\mathrm{FvW})$. Esta deficiencia genera la acumulación de multímeros extralargos de $\mathrm{FvW}$, lo que conlleva a la agregación plaquetaria y formación de microtrombos con oclusión de arteriolas y capilares, resultando en una isquemia orgánica, hemólisis microangiopática y trombocitopenia grave ${ }^{2,3}$.

Dentro de las causas secundarias que conducen a las microangiopatías trombóticas (MAT), se pueden encontrar múltiples condiciones como el síndrome HELLP, enfermedades autoinmunes como el lupus eritematoso sistémico, neoplasias, el uso de medicamentos antimaláricos, anti-agregantes plaquetarios e inmunosupresores y la infección por $\mathrm{VIH}^{2-4}$.

La infección por VIH puede generar manifestaciones hematológicas con importante repercusión en la morbimortalidad del paciente. Puede afectar las tres líneas celulares, siendo la más frecuente la anemia, linfopenia, neutropenia, y trombocitopenia, en ese orden ${ }^{10}$. La anemia más común es la secundaria a una enfermedad crónica, seguida de la asociada a compromiso por infiltración medular, citopenias inducidas por medicamentos, compromiso por infecciones oportunistas (como histoplasmosis o infección por parvovirus humano B19) o incluso afectación autoinmune (anemia hemolítica por anticuerpos calientes $)^{11,12}$. Por otro lado, el compromiso plaquetario en forma de trombocitopenia, puede alcanzar un 5-15\% al inicio de la enfermedad ${ }^{13}$.

La microangiopatía trombótica asociada al VIH se describió por primera vez en 1984 por el Dr. Boccia ${ }^{14}$. $\mathrm{Su}$ comportamiento epidemiológico ha variado debido a la introducción de la terapia anti-retroviral altamente efectiva (TARV), disminuyendo su incidencia desde $7,1 \%$ y una expectativa de vida menor de dos años, a una incidencia post-tratamiento de $0,3 \% \mathbf{0}^{13,15,16}$. En términos generales, los pacientes con infección por VIH tienen una incidencia de PTT entre 15 y 40 veces mayor que la población no infectada ${ }^{17,18}$.

Presentamos el caso de un paciente que debutó con una PTT como primera manifestación de la infección por VIH.

\section{Caso clínico}

Varón de 28 años, natural y procedente de Bogotá (Colombia), estudiante universitario, sin antecedentes mórbidos, que ingresó al servicio de urgencias con historia de una semana de evolución de fiebre, astenia y adinamia asociados a episodios de gingivorragia; sin diarrea $u$ otro síntoma gastrointestinal. Al ingreso se encontraba taquicárdico, febril, orientado. Al examen físico presentaba palidez mucocutánea y lesiones petequiales en el paladar.

Dentro de los exámenes de laboratorio del ingreso destacaba una hemoglobina de $6,9 \mathrm{mg} / \mathrm{dl}$, confirmando una anemia normocítica, normocrómica y una trombocitopenia intensa, con un recuento de plaquetas de 12.000 céls $/ \mathrm{mm}^{3}$. La función renal era normal, con una creatinina $0,7 \mathrm{mg} / \mathrm{dl}$, deshidrogenasa láctica elevada $702 \mathrm{UI} / \mathrm{ml}$, con bilirrubina y tiempos de coagulación normales. Por sospecha de hemólisis se realizó una prueba de Coombs directa que resultó negativa, reticulocitos: 11,9\% (corregido $4,87 \%$ ) y haptoglobina: $<8 \mathrm{mg} / \mathrm{dl}$ (consumida). Con la confirmación de una anemia hemolítica no inmune en un paciente sin antecedente familiar de hemoglobinopatía, se solicitó un extendido de sangre periférica, que demostró presencia de anisocitosis, con microcitos ++ , macrocitos y esquistocitos + ; trombocitopenia y anisocitosis plaquetaria y leucocitos normales.

Se descartaron causas infecciosas, con hemocultivos negativos y serologías para virus hepatotropos, hemoparásitos en sangre y pruebas no treponémicas para sífilis, todos negativos. La radiografía de tórax fue normal. No tenía antecedentes de valvulopatías ni prótesis cardíaca. Durante su estancia hospitalaria persistió con fiebre sin documentar infección clínica ni de laboratorio.

Dado lo anterior más los hallazgos de laboratorio se sospechó una microangiopatía trombótica, por lo que se solicitaron estudios de autoinmunidad: complemento C3 y C4 que fueron normales; anticuerpos antinucleares (ANAs), antiDNA, anticitoplasma de neutrófilos (ANCA), anticoagulante lúpico, IgG e IgM anti- $\beta 2$ glicoproteína e $\operatorname{IgG}$ e IgM anticardiolipinas, que resultaron todos negativos.

Por sospecha de una anemia microangiopática no autoinmune, se calculó el puntaje PLASMIC que dio 7 puntos, clasificándose como grupo de alto riesgo y $72 \%$ de riesgo para deficiencia grave en la actividad de la enzima ADAMTS 13. Se realizó estudio de citometría de flujo la cual fue negativa para hemoglobinuria paroxística nocturna (FLAER, CD157, CD15, CD64 y CD45) y negativa 


\begin{tabular}{|c|c|c|c|c|}
\hline Examen de laboratorio & Ingreso & Día+3 & Día+9 & Día+12 \\
\hline Hemoglobina (g/dl) & 6 & 7,6 & 8,9 & 11 \\
\hline Hematocrito (\%) & 18,4 & 23,3 & 28,4 & 33,9 \\
\hline VCM (fL) & 85,8 & 87 & 89,4 & 89,8 \\
\hline Plaquetas (céls/mm³) & 12.000 & 22.600 & 173.000 & 206.000 \\
\hline Leucocitos (céls/mm³) & 7.500 & 6.660 & 6.270 & 6.470 \\
\hline Neutrófilos (céls/mm³) & 4.200 & 4.760 & 3.660 & 3.270 \\
\hline LDH (Ul/ml) & 702 & 516 & 245 & 135 \\
\hline Bilirrubina total/indirecta $(\mathrm{mg} / \mathrm{dl})$ & $0,6 / 0,3$ & & & \\
\hline TP (seg) (pac/normal) & $17,9 / 15,1$ & & $14,5 / 15,1$ & \\
\hline INR & 1,18 & & 0,96 & \\
\hline TPT (seg) (pac/normal) & $38,5 / 30,9$ & & $31,2 / 30,9$ & \\
\hline Creatinina (g/dl) & 0,8 & 0,8 & 0,7 & \\
\hline BUN (mg/dl) & 12 & 19 & 17 & \\
\hline
\end{tabular}

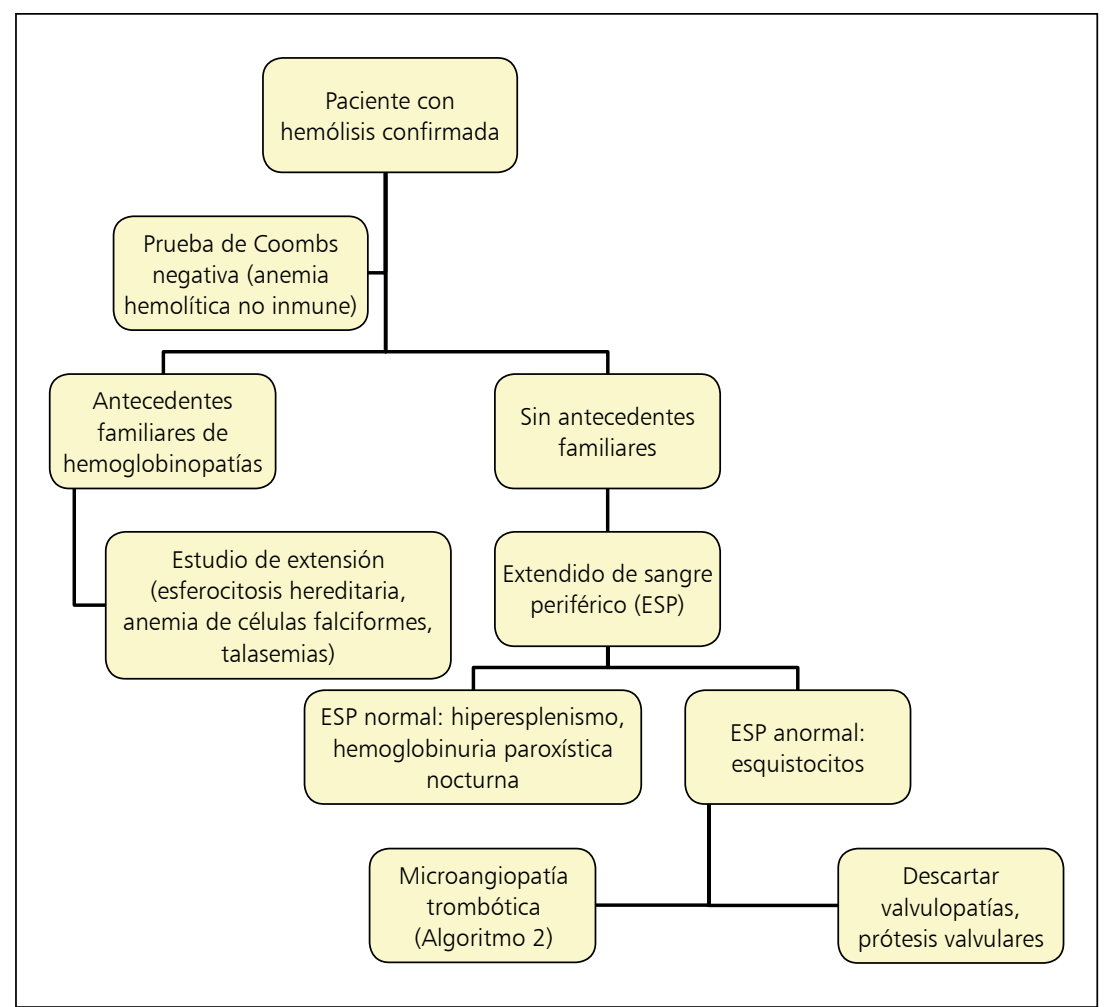

Figura 1. Algoritmo 1: diagnóstico de anemias hemolíticas no inmunes. Tomado y modificado de referencia bibliográfica 24 . para formas inmaduras (blastos), con niveles de vitamina B12 y ácido fólico normales.

Como parte del estudio de microangiopatía trombótica, con varias patologías infecciosas y autoinmunes descartadas, se solicitó una serología para VIH (ELISA de cuarta generación) que resultó positiva. La carga viral fue 69.777 copias $/ \mathrm{mm}^{3}$ y recuento de CD4: 167 céls $/ \mathrm{mm}^{3}$.

El paciente no cumplía criterios de síndrome retroviral agudo u otra manifestación que hiciera sospechar esta complicación. Dentro del estudio del paciente se descartó histoplasmosis con antígeno urinario de histoplasma negativo. Sin embargo, durante su estancia hospitalaria no se estudiaron serología para parvovirus humano B19, citomegalovirus y virus de Epstein Barr.

Posteriormente, se obtuvo el resultado de actividad de la enzima ADAMTS 13 en $6,8 \%$, confirmado con una segunda muestra. No se consideró SHUa, dado que la función renal durante toda su estancia hospitalaria fue normal, y nunca presentó síntomas gastrointestinales como para sospechar SHU. Con todos los resultados del estudio se diagnosticó una PTT asociada a VIH.

Durante su estancia en la unidad de cuidados intensivos recibió cinco sesiones de plasmaféresis, con mejoría en los recuentos celulares, resolución de la anemia y de la trombocitopenia (Tabla 1), por lo que fue posible el alta hospitalaria a las dos semanas del ingreso a la institución.

Según las guías de manejo nacionales de ese momento, se indicó terapia anti-retroviral con efavirenz/emtricitabina/tenofovir disoproxilo 600/200/245 mg, una vez al día. En el seguimiento ambulatorio, el paciente mantuvo un adecuado control, sin alteraciones hematológicas y con cargas virales de VIH indetectables.

\section{Discusión}

Presentamos el caso clínico de un paciente con infección por VIH, quien debutó con una PTT, sin otra manifestación que sugiriera la enfermedad retroviral, documentación de una infección oportunista o enfermedad definitoria de SIDA. Debido a la presencia de una anemia hemolítica no inmune, se aplicó un algoritmo diagnóstico inicial (Figura 1). Posteriormente, se buscaron causas identificables de una microangiopatía trombótica (Figura 2 ), descartando el uso de medicamentos, enfermedades autoinmunes e infecciones.

Por la sospecha diagnóstica y para el inicio temprano del tratamiento se utilizaron puntajes validados, como el PLASMIC score. Este fue desarrollado por Bendapudi y cols. ${ }^{19}$, basado en parámetros clínicos y de laboratorio obtenidos al ingreso del paciente. Se compone de siete elementos que son: el recuento plaquetario, la documentación de hemólisis, ausencia de cáncer activo o de trasplante de un órgano sólido, el volumen corpuscular medio (VCM), 


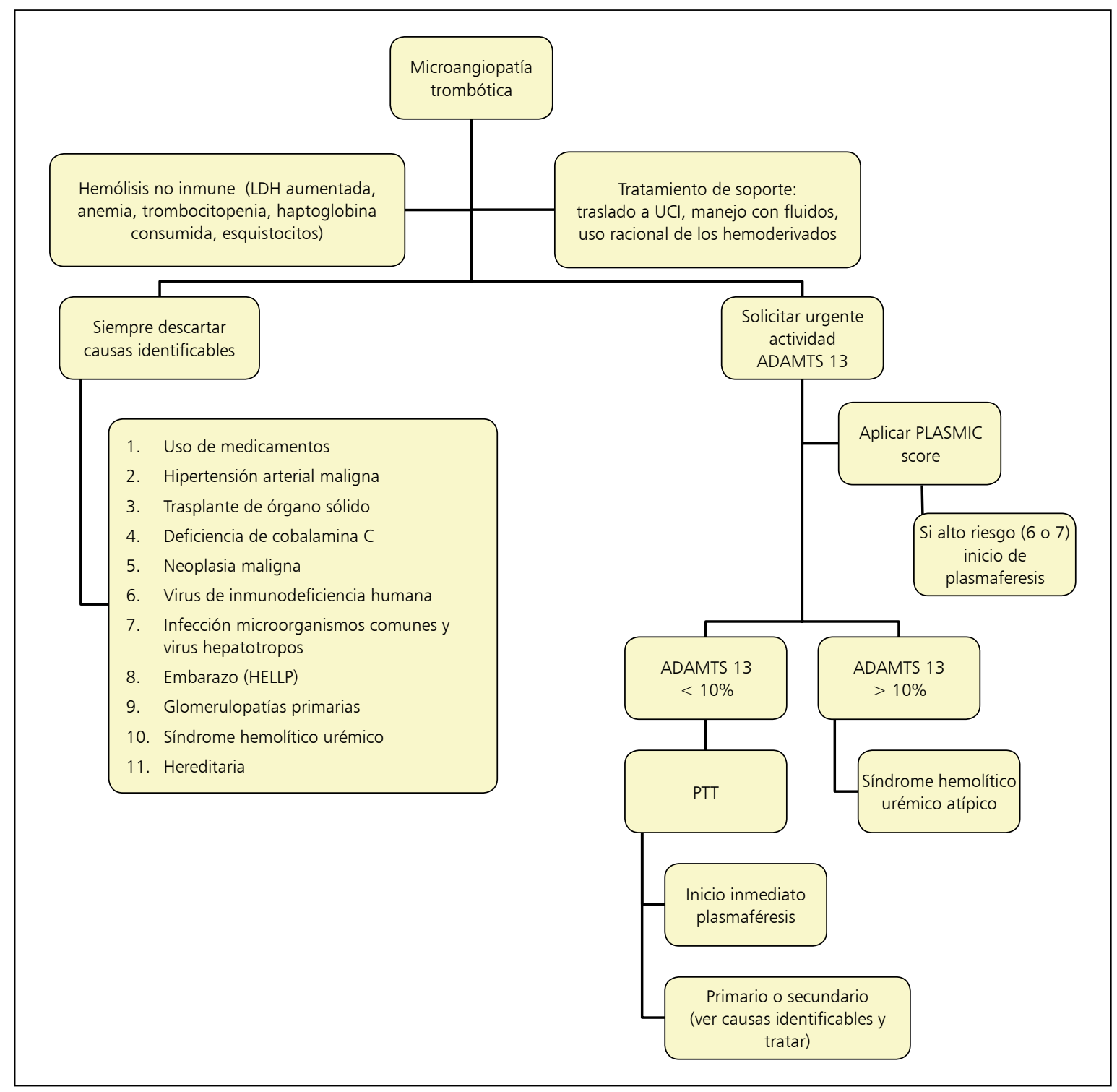

Figura 2. Algoritmo 2: aproximación diagnóstica de una microangiopatía trombótica. Tomado y modificado de referencias 2, 5, 21.
INR y la creatinina plasmática ${ }^{19,20}$. Tiene un puntaje de 0 a 7, con un punto de corte de 6 o 7 para alto riesgo para tener actividad de ADAMTS 13 menor al 10\%, con un área bajo la curva en la validación externa de $0,91^{19,21}$. Cumpliendo estos parámetros, se debe iniciar plasmaféresis en forma precoz en pacientes sin compromiso neurológico o elevación de biomarcadores cardíacos como la troponina I, en los cuales esta indicada esta terapia ${ }^{20}$. En el caso de nuestro paciente, con un puntaje de 7, se pudo iniciar tempranamente la plasmaféresis logrando la mejoría completa de las citopenias y un pronto egreso.

En los pacientes con infección por VIH que presentan microangiopatía trombótica, la enfermedad que más frecuentemente se presenta es PTT (70\%), seguida de SHU
$(30 \%)^{9}$. Algunos de los factores relacionados con su desarrollo son: recuentos bajos de CD4, carga viral elevada ${ }^{17,18}$ y presencia de infecciones oportunistas, las cuales se han asociado hasta en $89 \%$ de los $\operatorname{casos}^{15,17}$. Nuestro paciente tenía recuentos de CD4 disminuidos ${ }^{16-18,22,23}$ y carga viral elevada, apoyando de esta manera el diagnóstico de PTT. Sin embargo, no es frecuente esta presentación en el debut de la enfermedad (Tabla 2), más aún habiendo descartado infecciones oportunistas, como ocurrió en nuestro caso.

La patogénesis de la PTT se encuentra pobremente entendida ${ }^{15-17}$. Existen algunas teorías, como la desregulación de los linfocitos $\mathrm{T}$ sobre los linfocitos $\mathrm{B}$, permitiendo una producción de anticuerpos no controlada, con el potencial de generar anticuerpos dirigidos contra ADAMTS 


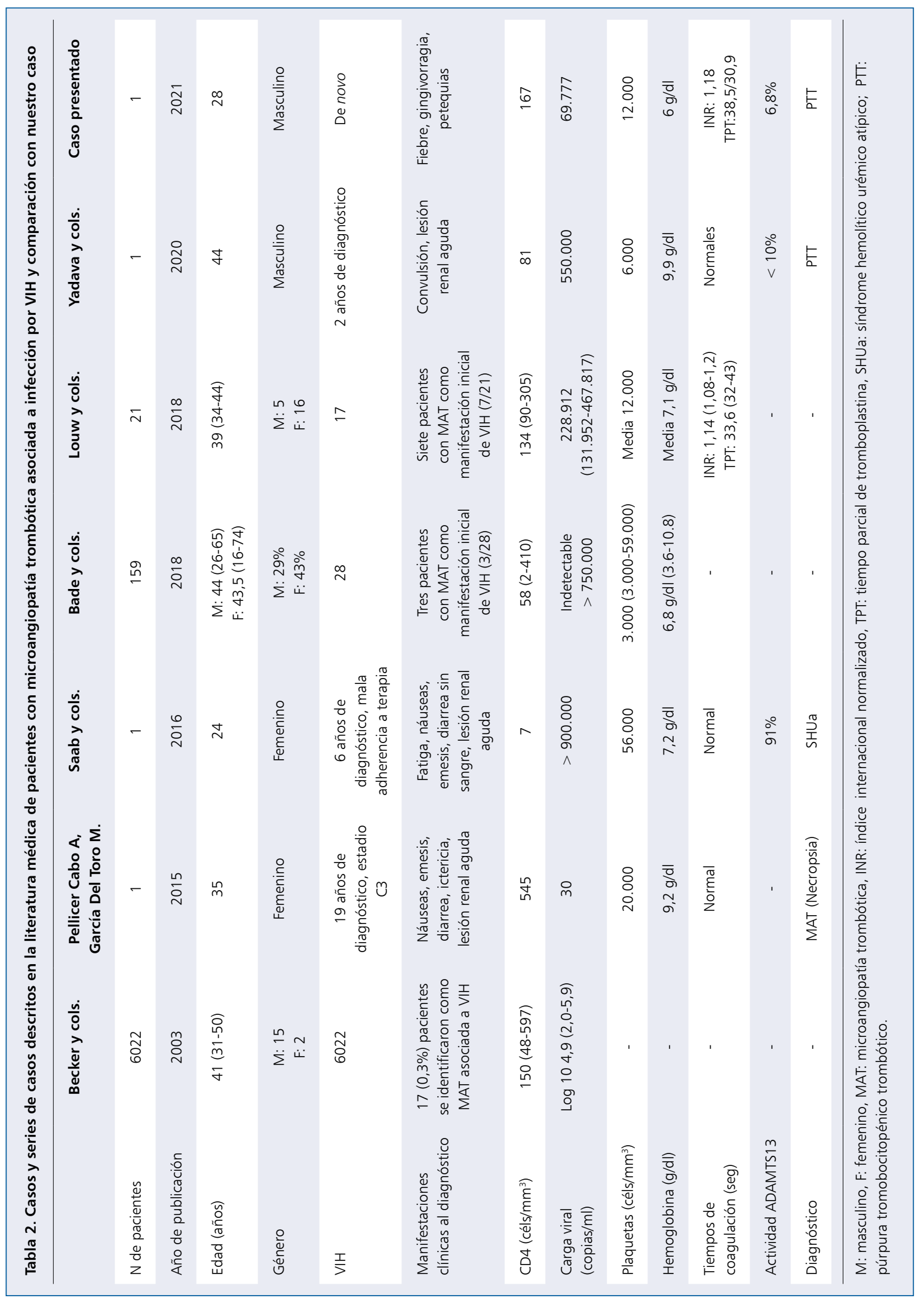


$\mathrm{dad}^{4}$, la que puede ser tan alta como $90 \%$ sin tratamien-

$13^{17,18,22} \mathrm{y}$, la teoría del compromiso directo del endotelio por el virus, lo que genera un aumento en la expresión de moléculas de adhesión como ICAM, VACM-1 y la E-selectina, promoviendo la adhesión leucocitaria y la liberación de multímeros de $\mathrm{FvW}^{15,17}$.

En cuanto al inicio de la TARV, no existe información al respecto cuando el paciente cursa con una microangiopatía trombótica asociado al VIH. La decisión de la terapia se debe dar por la presencia o no de infecciones oportunistas, según las guías internacionales de manejo de infección por $\mathrm{VIH}^{17}$.

El inicio del manejo con plasmaféresis con la sospecha de PTT, incluso previo al resultado, impacta en la letali- to $^{17,18}$. Sin embargo, esta no es una terapia libre de riesgos y complicaciones, por lo cual la decisión se debe apoyar en ciertos parámetros, como el score PLASMIC, el cual ha sido validado en países como el nuestro ${ }^{21}$.

En el contexto de la infección por VIH, el diagnóstico de PTT se convierte en un verdadero reto, dado que generalmente no se tiene en cuenta por su escasa frecuencia. Ante la sospecha diagnóstica, el uso de un algoritmo claro que contemple todas las posibles causas, y con la ayuda de scores validados, se puede llegar a un diagnóstico preciso y un manejo adecuado, con resultados clínicos favorables, como fue en nuestro caso.

\section{Referencias bibliográficas}

1.- Lammle B, Kremer Hovinga J A, Albeiro L. Thrombotic thrombocytopenic purpura J Thromb Haemost. 2005; 3: 1663-75. doi: 10.1111/j.1538-7836.2005.01425.x.

2.- Brocklebank V, Wood K M, Kavanagh D. Thrombotic microangiopathy and the kidney. Clin J Am Soc Nephrol. 2018; 13: 300-17. doi: 10.2215/CJN.00620117.

3.- Shenkman B, Einav Y. Thrombotic thrombocytopenic purpura and other thrombotic microangiopathic hemolytic anemias: Diagnosis and classification. Autoimmunity Reviews. Elsevier 2014; 13: 584-6.

4.- Tsai H M. Thrombotic thrombocytopenic purpura: Beyond empiricism and plasma exchange. Am J Med. 2019; 132: 1032-7. doi: 10.1016/j.amjmed.2019.03.009.

5.- Joly B S, Coppo P, Veyradier A. Thrombotic thrombocytopenic purpura. Blood 2017; 129 : 2836-46. doi: 10.1182/blood-2016-10-709857.

6.- Mariotte E, Azoulay E, Galicier L, Rondeau E, Zouiti F, Boisseau P, et al. Epidemiology and pathophysiology of adulthood-onset thrombotic microangiopathy with severe ADAMTS13 deficiency (thrombotic thrombocytopenic purpura): A cross-sectional analysis of the French national registry for thrombotic microangiopathy. Lancet Haematol. 2016; 3: e237-45. doi: 10.1016/S2352-3026(16)30018-7.

7.- $\quad$ Hamed N A M. Diagnosis and management of thrombotic thrombocytopenic purpura. J Blood Disord Symptoms Treat. 2018; 2: 1-6.

8.- Page E E, Kremer Hovinga J A, Terrell D R, Vesely S K, George J N. Thrombotic thrombocytopenic purpura: diagnostic criteria, clinical features, and long-term outcomes from 1995 through 2015. Blood Adv. 2017; 1: 590600. doi: 10.1182/bloodadvances.2017005124.

9.- Kalpatthi R, Kiss J E. Thrombotic thrombocytopenic purpura, heparin-induced thrombocytopenia, and disseminated intravascular coagulation. Crit Care Clin. 2020; 36: 357-77. doi: 10.1016/j.ccc.2019.12.006.

10.- Enberg M, Yaquich P, Pilasi C, Thompson L, Oddó D, Bronfman L. Anemia hemolítica autoinmune en paciente infectado por virus de inmunodeficiencia humana y enfermedad de Castleman. Rev Chilena Infectol. 2002; 19: 231-6. doi: 10.4067/S071610182002000400004.

11.- Parinitha S S, Kulkarni M H. Haematological changes in HIV infection with correlation to CD4 cell count. Australas Med J. 2012; 5: 15762. doi: 10.4066/AMJ.20121008.

12.- Choi S Y, Kim I, Kim N J, Lee S A, Choi Y A, Bae J Y, et al. Hematological manifestations of human immunodeficiency virus infection and the effect of highly active anti-retroviral therapy on cytopenia. Korean J Hematol. 2011; 46: 2537. doi: 10.5045/kjh.2011.46.4.253.

13.- Nascimento F G, Tanaka P Y. Thrombocytopenia in HIV-infected patients. Indian J Hematol Blood Transfus. 2012; 28 109-11. doi: 10.1007/s12288-011-0124-9.

14.- Boccia R V, Gelmann E P, Baker C C, Marti G, Longo D L. A hemolytic-uremic syndrome with the acquired immunodeficiency syndrome. Ann Intern Med. 1984; 101: 716-7. doi: 10.7326/0003-4819-101-5-716 2.

15.- Saab K R, Elhadad S, Copertino D, Laurence J. Thrombotic microangiopathy in the setting of HIV infection: a case report and review of the differential diagnosis and therapy. AIDS Patient Care STDS 2016; 30: 359-64. doi: 10.1089/ apc.2016.0124.

16.- Pellicer Cabo Á, García Del Toro M. Microangiopatía trombótica en una paciente con infección por el virus de la inmunodeficiencia humana. Infectio. 2015; 19 : 168-71. doi: 10.1016/j.infect.2014.12.004.

17.- Bade N A, Giffi V S, Baer M R, Zimrin A B, Law J Y. Thrombotic microangiopathy in the setting of human immunodeficiency virus infection: high incidence of severe thrombocytopenia. J Clin Apher. 2018; 33: 3428. doi: 10.1002/jca.21615.

18.- Louw S, Gounden R, Mayne E S. Thrombotic thrombocytopenic purpura (TTP)-like syndrome in the HIV era. Thromb J. 2018;16: 35. doi: 10.1186/s12959-018-0189-x.

19.- Bendapudi P K, Hurwitz S, Fry A, Marques M B, Waldo S W, Li A, et al. Derivation and external validation of the PLASMIC score for rapid assessment of adults with thrombotic microangiopathies: a cohort study. Lancet Haematol. 2017; 4: e157-64. doi: 10.1016/ S2352-3026(17)30026-1.

20.- Jamme M, Rondeau E. The PLASMIC score for thrombotic thrombocytopenic purpura. Lancet Haematol. 2017; 4: e148-9. doi: 10.1016/ S2352-3026(17)30024-8.

21.- Sousa Oliveira D, Lima T G, Neri Benevides F L, Tavares Barbosa S A, Oliveira M A, Boris $\mathrm{N}$ P, et al. Plasmic score applicability for the diagnosis of thrombotic microangiopathy associated with ADAMTS13-acquired deficiency in a developing country. Hematol Transfus Cell Ther. 2019; 41: 119-124. doi:10.1016/j.htct.2018.10.002.

22.- Becker S, Fusco G, Fusco J, Balu R, Gangjee $\mathrm{S}$, Brennan C, et al. HIV-associated thrombotic microangiopathy in the era of highly active antiretroviral therapy: an observational study. Clin Infect Dis. 2004; 39(S5): S267-75. doi: $10.1086 / 422363$.

23.- Yadava S K, Vyas V, Jain H, Fazili T. Thrombotic thrombocytopenic purpura associated with AIDS. AIDS 2020; 34 : 1101-2.

24.- González Mesones B, González De Villambrosia A, Batlle A, Insunza A. Protocolo diagnóstico de las anemias hemolíticas. Medicine 2012; 11: 1246-9. doi: 10.1016/ S0304-5412(12)70477-8. 